\title{
Interference-Aware Channel Assignment Algorithm in D2D overlaying Cellular Networks
}

\author{
Liqun Zhao ${ }^{1}$, Hongpeng Wang ${ }^{1}$ and Xiaoxiong Zhong ${ }^{2,3,4}$ \\ ${ }^{1}$ School of Computer, Harbin Institute of Technology (Shenzhen) \\ Shenzhen, Guangdong, 518055, China \\ [e-mail: lxzlq2005@126.com, wanghp@hit.edu.cn] \\ ${ }^{2}$ Peng Cheng Laboratory, Shenzhen 518000, China \\ ${ }^{3}$ Graduate School at Shenzhen, Tsinghua University, Shenzhen 518055, P. R. China \\ ${ }^{4}$ Guangxi Key Laboratory of Trusted Software, Guilin University of Electronic Technology \\ Guilin, Guangxi, 541004, China \\ [e-mail: xixzhong@gmail.com] \\ *Corresponding authors: Liqun Zhao, Xiaoxiong Zhong
}

Received July 19, 2018; revised September 15, 2018; accepted September 30, 2018; published April 30, 2019

\begin{abstract}
Device-to-Device (D2D) communications can provide proximity based services in the future 5G cellular networks. It allows short range communication in a limited area with the advantages of power saving, high data rate and traffic offloading. However, D2D communications may reuse the licensed channels with cellular communications and potentially result in critical interferences to nearby devices. To control the interference and improve network throughput in overlaid D2D cellular networks, a novel channel assignment approach is proposed in this paper. First, we characterize the performance of devices by using Poisson point process model. Then, we convert the throughput maximization problem into an optimal spectrum allocation problem with signal to interference plus noise ratio constraints and solve it, i.e., assigning appropriate fractions of channels to cellular communications and D2D communications. In order to mitigate the interferences between D2D devices, a cluster-based multi-channel assignment algorithm is proposed. The algorithm first cluster D2D communications into clusters to reduce the problem scale. After that, a multi-channel assignment algorithm is proposed to mitigate critical interferences among nearby devices for each D2D cluster individually. The simulation analysis conforms that the proposed algorithm can greatly increase system throughput.
\end{abstract}

Keywords: D2D, cellular network, 5G, channel assignment, interference management.

A preliminary version of this paper appeared in 10th International Conference on Wireless Internet (WICON) 2017, December 16-17, Tianjin, China. This version includes a novel cluster-based multi-channel algorithm to mitigate the critical interferences from nearby D2D devices and further improve network throughput. This research was supported by Shenzhen IOT key technology and application systems integration engineering laboratory and the the National Natural Science Foundation of China under grant 61802221 and the Natural Science Foundation of Guangxi Province under grant 2016GXNSFBA380010, and 2017GXNSFAA198192. 


\section{Introduction}

Local area services based on content sharing, such as live streaming, file distribution, environment monitoring, etc., have been witnessed quick growth in recent years. By adopting D2D communications in cellular networks, proximate devices are allowed to communicate directly without forwarded by the base station (BS) [1][2]. Although D2D communications bring multiple advantages, such as, shorter delay, energy saving, higher transmission rate, traffic offloading, etc., it results in complicated interference problem in a cell, which could be deteriorating network performance [3]. How to mitigate the interference among nearby devices is still a challenge to improve network performance [4].

The technology of orthogonal frequency division multiplexing (OFDM) has been applied to LTE cellular network to improve system performance. The flexible multi-channel assignment provides various QoS (Quality of Service) to different services, i.e., more than one OFDM channels can be assigned to a typical user equipment (UE) or device to satisfy the QoS requirements. This makes the channel assignment problem more complicated.

Usually, there are two categories of D2D communication in cellular networks according to spectrum allocation mode, i.e., in-band D2D and out-band D2D. For in-band D2D, the D2D communications rely on the licensed spectrum resource of network, which increases the channel reuse factor and spectrum efficiency. However, the serious interferences among nearby devices could be generated. In-band D2D can work in underlay mode if D2D communications reuse the same channels as cellular communications or in overlay mode if D2D communications and cellular communications use different fractions of system channels. The most obvious advantage of underlay D2D is the higher spectrum efficiency since a channel can be shared by both cellular communication and D2D communications. However, interferences are generated from DUEs (D2D user equipments) to CUEs (cellular user equipments) and the cellular communication may be deteriorated. When compared to underlaid cellular networks, the interference scenario in overlaid cellular networks is simpler since there is no interference generated between CUEs and DUEs. The interferences among DUEs is relatively lower due to the limited transmit power of UEs. In case of out-band D2D, the D2D link can use the unlicensed spectrum of other network, such as WiFi and Bluetooth and result in uncontrollable interference.

The interference is the most critical problem in cellular networks and attracts the attention of research communities [5][6]. Many algorithms which focus on interference mitigation and channel assignment in underlaid D2D cellular networks have been proposed [7][8][9][10][11][12][13]. Power control is the most simple and straightforward method to control the interference among devices. In [7], an optimal power control algorithm is proposed to maximize the sum-rate of devices. Although reducing transmit power can alleviate the suffered interference at a device, the communication distance is also reduced at the same time. Intelligent channel assignment is an efficient way to improve network performance by assigning appropriate channels to devices. In [8], the authors defined an interference to signal plus ratio (ISR) threshold to D2D receiver. If the ISR of a DUE greater than the threshold, the DUE cannot share any channels with CUEs and the severe interference from DUE to CUE is subsequently avoided. In order to mitigation interferences in cellular networks, some works collect some extra information (e.g., path loss, locations of UEs, shadowing, channel state 
information etc.) to design channel assignment algorithms [9][10]. In these algorithms, a cellular device and a D2D device cannot share any channels according to their exact locations or the distances between devices and BS. In [11], the authors combine power allocation and mode selection design a channel assignment to satisfy the QoS requirements of devices. In [12], the authors propose a novel channel assignment algorithm which minimizes the interferences among small cells, D2D communications and cellular communications in a heterogeneous cellular network. On the other hand, there a lot of works focus on the D2D overlaid cellular network or only allow part of D2D communications share channels with cellular communications. In [13], the authors research the performance of D2D relaying cellular network using stochastic geometry. In [14], the authors propose a multi-channel based scheduling algorithm. The algorithm schedules communications according to a SINR-aware priority. Simulation results show that the proposed algorithm outperformed the single one-channel-based algorithm. In [15], the authors propose a mode selection algorithm which combines the advantages of both underlay mode and overlay mode. In the algorithm, some devices can switch to overlay mode to improve the spectrum utility. In [16], the authors propose a social-community-aware D2D resource allocation algorithm. In the algorithm, cellular communications and D2D communications with close social ties are allowed to reuse the same channels, otherwise cellular communications and D2D communication cannot share any channels. Some works focus on the cooperative transmissions based channel sharing in the overlaid/underlaid cellular networks. In [17], a contract-based cooperative channel sharing algorithm is proposed to exploit the transmission opportunities for the D2D communications and keep the maximum profit of the cellular communications. Although a lot of algorithms have been taken on interference mitigation in D2D enhanced cellular networks, there is still room for improvement. First, most of the current algorithms have high computational complexity and overhead incurred in coordination, extra information collection, etc. It is difficult to apply these algorithms to current cellular networks due to the large cost of upgrading despite their performance gain [18]. Second, some works only considered some specific cases of network, such as the fixed D2D communication distance, allowing only one cellular communication and one D2D communication reuse a channel, etc. Third, some works only mitigate the interferences within a single cell, the interferences from adjacent cells are not considered. Forth, some works only focus on the single channel assignment, which cannot be applied to multi-channel based systems, such as LTE. At last, the majority of the existing works are focus on the hexagonal grid structure, which is too simple to represent the locations of devices and BSs [19]. In the past few years, Poisson point process (PPP) model has become a universal mathematical tool to modeling the locations of base stations and devices. It has been proven to be accurate in terms of signal to interference plus noise ratio (SINR) distribution when compared to hexagonal grid model [20, 21].

Part of this work has been published in [22], where optimal spectrum allocation algorithm is proposed to maximize the network throughput on the condition that D2D communications can randomly select channels from a specific fraction of system channels. Unfortunately, the interference problem among nearby DUEs is not addressed. In this work, we propose a novel cluster-based multi-channel algorithm to further mitigate the interference from DUEs.

In this paper, a novel channel assignment approach is proposed to control interference in overlaid D2D cellular networks. Two main problems are addressed in this paper: How to assign appropriate fractions of channels to cellular communications and D2D communications? How to assign appropriate channels to D2D communications? For the first problem, we first 
derive the outage probability and ergodic rate to analyze the throughput of CUE and DUE respectively. After that, a novel spectrum allocation algorithm is proposed to control network interference and improve system throughput. For the second problem, a cluster-based multi-channel assignment algorithm is proposed to mitigate the critical interferences among nearby D2D devices. The main contributions of this paper are summarized as follows:

(1) We consider a multi-channel setting in cellular communications and D2D communications. Therefore, the proposed algorithm can be applied to OFDM based cellular communication systems, such as LTE and LTE-A.

(2) We introducing a novel spectrum allocation algorithm to improve the system throughput. We first model the locations of UEs and BSs by PPP model. Then we derive the UE outage probability and ergodic rate to find the relationship between spectrum allocation and UE throughput. At last, we convert the throughput maximization problem into an optimal spectrum allocation problem with SINR constraints and assign appropriate fractions of channels to cellular communications and D2D communications.

(3) A novel cluster-based multi-channel assignment algorithm is proposed to mitigate the critical interferences among D2D devices. First, we divide D2D communications into clusters according to their potential interference relationships. Then, we present a multi-channel assignment algorithm which assigns channels to D2D communications to minimize the interference in each D2D cluster.

(4) We make extensive simulations on multi-cell scenario and the results show that the network performance is further improved.

The rest part of this paper is organized as follows. Section 2 introduces the D2D overlaid cellular network model and assumptions used throughout this paper. In section 3, we analyze the network characters by using PPP model and assign appropriate fraction channels to cellular communications and D2D communications. The proposed cluster-based multi-channel assignment algorithm is described in section 4. Simulation results and analyses are given in section 5. Finally, conclusions will be made in section 6 .

\section{System Model}

A multi-cell overlaid cellular network is considered as shown in Fig. 1. We define CUE as the device which communication with BS and DUE as a device which direct communication with another device by D2D. Two types of links exist in our network: links between BS and CUE are direct links and links between DUEs are D2D links. A D2D communication contains two DUEs (i.e. a D2D transmitter and a D2D receiver) in which two DUEs communicate directly. The locations of BSs follow a homogeneous PPP $\Phi_{b}$ with density $\lambda_{b}$, the locations of CUEs and DUE transmitters are modeled as another PPP with density $\lambda_{c}$ and $\lambda_{d}$ that are independent of $\Phi_{b}$. The communication distance between two DUEs in a D2D pair follows a uniformed distribution on interval $(0, b)$. The transmit power of BS and DUEs are denoted by $P_{b}$ and $P_{D}$. $\alpha_{C}$ and $\alpha_{D}$ are the path loss exponents for direct link and D2D link. We also assume that system is full load and the system channels are always fully occupied by UEs. 


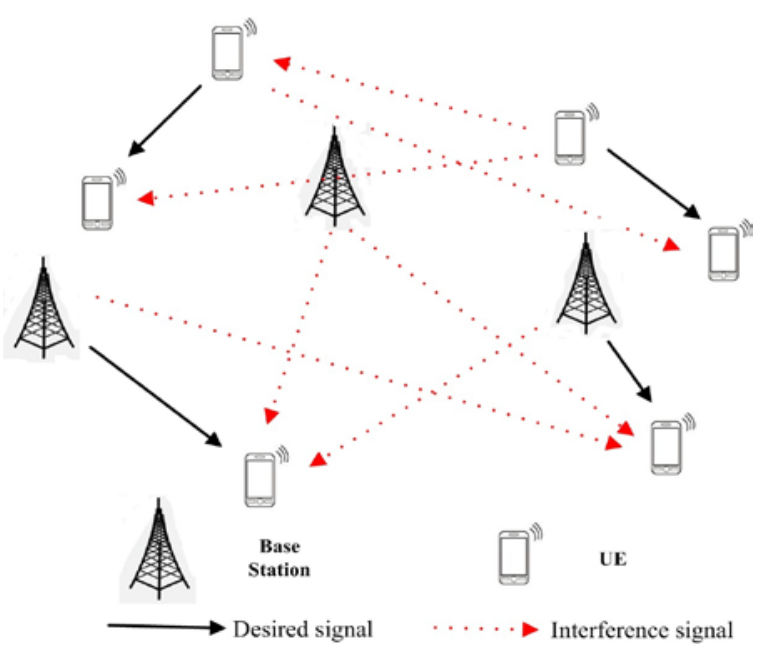

Fig. 1. System model.

As shown in Fig. 2, the overall spectrum resource are divided into $N$ orthogonal channels and the bandwidth of each channel is $k \mathrm{~Hz}$. A spectrum allocation approach is considered in which DUE communications are active on $\eta N$ channels in the frequency domain. The rest $(1-\eta) N$ channels are occupied by cellular communications. Therefore, no interference exists between CUE and DUE. We also assume that each D2D pair randomly selects $N_{D}$ channels in $\eta N$ independently. Note that if it has $\eta N=N_{D}$, all D2D communications share the same channels. If it has $\eta N>N_{D}$, each DUE randomly selects $N_{D}$ channels form $\eta N$ channels. Then, the assigned channels of D2D communications are not always identical and the interferences between D2D communications can be reduced.

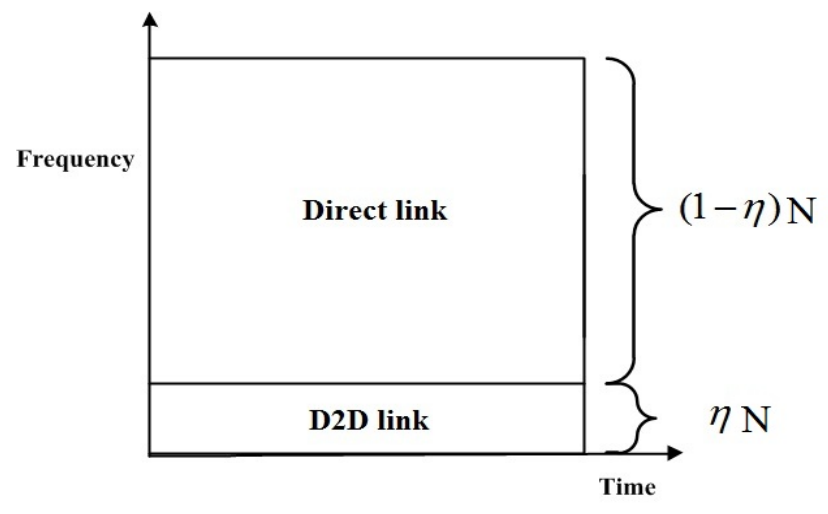

Fig. 2. Frame structure.

\section{Optimal Spectrum Allocation}

In this section, we convert the throughput maximization problem into an optimal spectrum allocation problem. To model the problem, we need to derive the expressions of outage probability and average ergodic rate for UEs. 


\subsection{Association probability}

We assume that each CUE select BS with the maximum received power. The probability that a typical UE is associated with BS can be expressed as

$$
A_{C}=\frac{\lambda_{C}}{\lambda_{C}+\lambda_{D}}
$$

The probability that a typical link belongs to D2D communication can be expressed as

$$
A_{D}=\frac{\lambda_{D}}{\lambda_{C}+\lambda_{D}}
$$

The average number of CUEs in a BS can be calculated as

$$
N_{C U E}=\frac{\lambda_{C}}{\lambda_{b}}
$$

\subsection{Outage probability}

In our work, the outage probability of a typical UE is defined as the probability that the received SINR is lower than a prescribed SINR threshold on each channel.

Lemma 1 If $T_{C}$ denote the prescribed SINR threshold of CUE, the outage probability of a typical CUE can be calculated as

$$
\mathbb{P}\left[\operatorname{SINR}_{C}<T_{C}\right]=1-2 \pi \lambda_{b} \int_{0}^{\infty} x \exp \left\{-\frac{T_{C}}{S N R_{C}}-\pi x^{2} \lambda_{b}\left[1+\mathcal{Z}\left(T_{C}, \alpha_{C}, 1\right)\right]\right\} d x
$$

where $\mathcal{Z}\left(T_{C}, \alpha_{C}, 1\right)=T_{C}^{\frac{2}{\alpha_{C}}} \int_{\left(\frac{1}{T_{C}}\right)^{2 / \alpha_{C}}}^{\infty} \frac{1}{1+t^{\alpha_{C} / 2}} d t$.

\section{Proof:}

Let $x$ be the distance between CUE and its serving BS, we have

$$
\mathbb{P}\left[\operatorname{SINR}_{C}<T_{C}\right]=1-\int_{0}^{\infty} \mathbb{P}\left[\operatorname{SINR}_{C}>T_{C}\right] f_{C}(x) d x
$$

The probability density function (PDF) of $x$ is

$$
f_{C}(x)=e^{-\pi \lambda_{b} x^{2}} 2 \pi \lambda_{b} x
$$

This is because the locations of BSs follows homogeneous $\operatorname{PPP} \Phi_{b}$ with density $\lambda_{b}$ in area $S$, its cumulative distribution function (CDF) is $\exp \left(-\lambda_{b} S\right)$.

In order calculate $\mathbb{P}\left[S I N R_{C}>T_{C}\right]$, we first calculate the suffered interferences for a typical CUE. As Fig. 3 shows, we assume that a CUE locates at the origin and its serving BS is $b_{0}$. The suffered interferences come from the BSs except $b_{0}$ which follow PPP $\Phi_{b}$ with density $\lambda_{b}$. Then, the suffered interferences can be expressed as

$$
I_{C}=P_{b} \sum_{\Phi_{b} \backslash b_{0}} H_{x} d_{C}^{-\alpha_{C}}
$$


where $d_{C}$ denote the distance between the typical CUE and interfering BSs. $H_{x}$ represents the channel gain. According to [23], the Laplace transform of $I_{C}$ can be calculated as

$$
\begin{aligned}
\mathcal{L}_{I_{C}}(s) & =E_{I_{C}}\left[\exp \left(-s I_{C}\right)\right] \\
& =E_{\Phi_{b}}\left[\exp \left(-s P_{b} \sum_{\Phi_{b} \backslash b_{0}} H_{x} d_{C}^{-\alpha_{C}}\right)\right] \\
& =\exp \left(-2 \pi \lambda_{b} \int_{z}^{\infty}\left\{1-\mathcal{L}_{H_{x}}\left(s P_{b} r^{-\alpha_{C}}\right)\right\} r d r\right) \\
& =\exp \left(-2 \pi \lambda_{b} \int_{z}^{\infty} \frac{r}{1+\left(s P_{b}\right)^{-1} r^{\alpha_{C}}} d r\right) \\
& =\exp \left(-2 \pi \lambda_{b} \int_{z}^{\infty} \frac{s P_{b} r}{s P_{b}+r^{\alpha_{C}}} d r\right)
\end{aligned}
$$

where $z$ detotes the distance between typical CUE and its closest interfering BS and

$$
\begin{aligned}
\mathbb{P}\left[S I N R_{C}>T_{C}\right] & =\mathbb{P}\left[P_{b} H_{x} x^{-\alpha_{C}} / I_{C}+\sigma^{2}>T_{C}\right] \\
& =\mathbb{P}\left[H_{x}>x^{\alpha_{C}} P_{b}^{-1} T_{C}\left(I_{C}+\sigma^{2}\right)\right] \\
& =\exp \left\{-\frac{T_{C}}{S N R_{C}}\right\} \mathcal{L}_{I_{C}}\left(x^{\alpha_{C}} P_{b}^{-1} T_{C}\right)
\end{aligned}
$$

where $S N R_{C}$ is the signal to noise ratio (SNR) of CUE and $S N R_{C}=\frac{P_{b} x^{-\alpha_{C}}}{\sigma^{2}}$. Combing (8) and (9), we have

$$
\mathbb{P}\left[\operatorname{SINR}_{C}>T_{C}\right]=\exp \left\{-\frac{T_{C}}{S N R_{C}}\right\} \exp \left(-2 \pi \lambda_{b} \int_{d}^{\infty} \frac{T_{C} X^{\alpha_{C}} r}{T_{C} \chi^{\alpha_{C}}+r^{\alpha_{C}}} d r\right)
$$

Let $t=x^{-2} r^{2} T_{C}^{-2 / \alpha_{C}}$, we get

$$
\mathbb{P}\left[\operatorname{SINR}_{C}>T_{C}\right]=\exp \left\{-\frac{T_{C}}{S N R_{C}}-\pi \lambda_{b} \mathcal{Z}\left(T_{C}, \alpha_{C}, 1\right) x^{2}\right\}
$$

where $\mathcal{Z}\left(T_{C}, \alpha_{C}, 1\right)=T_{C}^{\frac{2}{\alpha_{C}}} \int_{\left(\frac{1}{T_{C}}\right)^{2 / \alpha_{C}}}^{\infty} \frac{1}{1+t^{\alpha_{C} / 2}} d t$.

Plugging (5), (6) into (11) gives the desired result in (4). Note that the outage probability is independent of $\lambda_{b}$ and $P_{b}$. This property is same as [24]. 


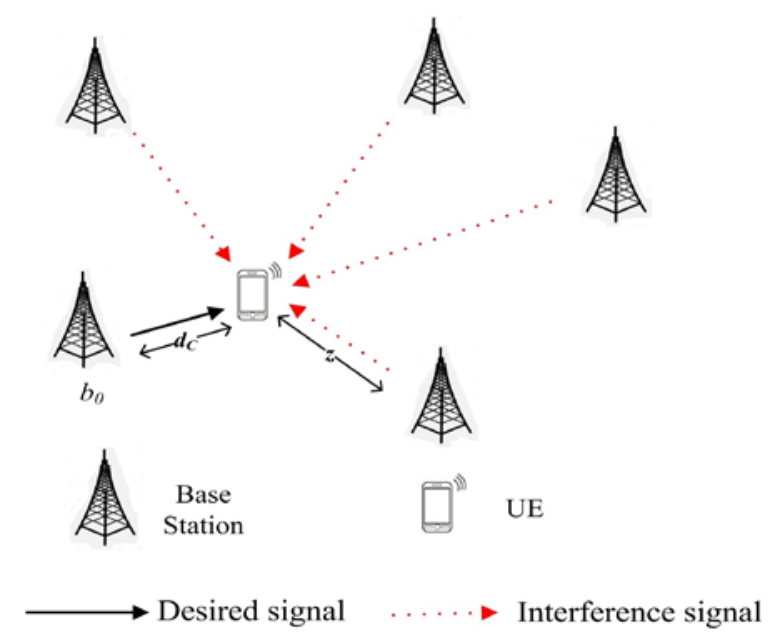

Fig. 3. The suffered interferences of a typical CUE.

Lemma 2 If $T_{D}$ denote the prescribed SINR threshold of D2D communication, the outage probability can be calculated as

$$
\mathbb{P}\left[\operatorname{SINR}_{D}<T_{D}\right]=1-\frac{1}{b} \int_{0}^{b} \exp \left\{-\frac{T_{D}}{S N R_{D}}-\pi N_{D} \lambda_{D} \mathcal{Z}\left(T_{D}, \alpha_{D}, 1\right) x^{2} / \eta N\right\} d x
$$

\section{Proof:}

Let $r$ be the distance of D2D communication, the PDF of $r$ is

$$
f_{d}(r)=1 / b
$$

This is because $r$ follows a uniformed distribution on interval $(0, b)$.

In a dense D2D overlaid cellular network, a large number of D2D communications exist in the network. When a DUE suffers multiple interferences from D2D transmitters on the same channels simultaneously, the achievable SINR can be significantly reduced. According to the network settings, the interference for a DUE receiver is from other D2D communications which share the same channel. The probability that two D2D communications occupy the same channels is $N_{\mathrm{D}} / \eta_{N}$.

The process of deriving DUE outage probability is similar to CUE. This is because a DUE suffers interferences from the D2D transmitters with density $\lambda_{D} N_{D} / \eta N$. By using a similar mathematical derivation, the access probability of D2D communications can be calculated as

$$
\mathbb{P}\left[\operatorname{SINR}_{D}>T_{D}\right]=\int_{0}^{b} \exp \left\{-\frac{T_{D}}{S N R_{D}}-\pi \lambda_{D} N_{D} \mathcal{Z}\left(T_{D}, \alpha_{D}, 1\right) x^{2} / \eta N\right\} d x
$$

Thus, the outage probability of D2D communication can be calculated as

$$
\mathbb{P}\left[\operatorname{SINR}_{D}<T_{D}\right]=1-\int_{0}^{b} \mathbb{P}\left[\operatorname{SINR}_{D}>T_{D}\right] f_{D}(x) d x
$$

Combing (13), (14) and (15), we get the desired result in (12). 
3.3 Average ergodic rate

Lemma 3 If each BS assigns equal channels to cellular links (i.e. Round Robin algorithm is adopted). The average ergodic rate of CUE can be expressed as

$$
R_{c}=2 \pi \lambda_{b} \int_{r>0} \int_{t>0} \exp \left\{-\frac{e^{t}-1}{S N R_{C}}-\pi \lambda_{b} r^{2}\left[\mathcal{Z}\left(e^{t}-1, \alpha, 1\right)+1\right]\right\} r d t d r
$$

\section{Proof:}

The average ergodic rate of a CUE $R_{C}$ is defined as the data rate average over the communication distance $x$ when all cell channels allocated to that CUE. According to Shannon's theory, we have

$$
R_{C}=E_{x}\left[E_{\operatorname{SINR}_{C}}\left[\ln \left(1+\operatorname{SINR}_{C}(x)\right)\right]\right]
$$

where

$$
\begin{aligned}
E_{\text {SINR }_{C}}\left[\ln \left(1+\operatorname{SINR}_{C}(x)\right)\right] & =\int_{0}^{\infty} \mathbb{P}\left[\ln \left(1+\operatorname{SINR}_{C}(x)\right)>t\right] d t \\
& =\int_{0}^{\infty} \exp \left\{-\frac{e^{t}-1}{\operatorname{SNR}_{C}}\right\} \mathcal{L}_{I_{C}}\left(x^{\alpha_{C}} P_{b}^{-1}\left(e^{t}-1\right)\right) d t \\
& =\int_{0}^{\infty} \exp \left\{-\frac{e^{t}-1}{S N R_{C}}-\pi \lambda_{b} x^{2} \mathcal{Z}\left(e^{t}-1, \alpha_{C}, 1\right)\right\} d t
\end{aligned}
$$

Plugging (18) to (17), we have

$$
R_{C}=\int_{0}^{\infty} E_{\text {SINR }_{C}}\left[\ln \left(1+\operatorname{SINR}_{C}(x)\right)\right] f_{c}(x) d x
$$

By combing (6) and (19), we get (16). By following a similar mathematical derivation process, the average ergodic rate of a D2D communication can be calculated as

$$
\begin{aligned}
R_{D} & =\int_{0}^{\infty} E_{S I N R_{D}}\left[\ln \left(1+\operatorname{SINR}_{D}(x)\right)\right] f_{D}(x) d x \\
& =\frac{1}{b} \int_{0}^{b} \int_{0}^{\infty} \exp \left\{-\frac{e^{t}-1}{S N R_{D}}-\frac{\lambda_{D} N_{D} r^{2}}{\eta N} \mathcal{Z}\left(e^{t}-1, \alpha_{D}, 1\right)\right\} d t d r
\end{aligned}
$$

where $S N R_{D}$ is the SNR for DUE receiver and $S N R_{D}=\frac{P_{D} x^{-\alpha_{D}}}{\sigma^{2}}$. Note that the average ergodic rates of D2D communications determined by the parameter $\eta$ which represents the number of channels assigns to D2D communications. This demonstrates that the reasonable channel allocation can increase the system performance.

\subsection{Optimal spectrum allocation}

In this section, we convert the throughput maximization problem into an optimal spectrum allocation problem. Our objective is maximizing the network throughput by finding an optimal parameter $\eta$. The cell throughput can be the sum throughput of CUE and DUE which are determined by spectrum allocation parameter $\eta$. Since the average ergodic rate of CUE has been derived in (20), the throughput of CUE can be calculated as 


$$
T_{C U E}=\mathbb{P}\left[\operatorname{SINR}_{C}>T_{C}\right] R_{c} \frac{(1-\eta) N k}{N_{C U E}}
$$

where $(1-\eta) N / N_{\text {CUE }}$ is the number of channels which allocated to each CUE. The throughput of D2D communications is

$$
T_{\text {DUE }}=\mathbb{P}\left[\operatorname{SINR}_{D}>T_{D}\right] R_{D} N_{D} k .
$$

Combing (21) and (22), we can calculate the throughput which is the sum throughput of CUEs and DUEs, that is

$$
\begin{aligned}
T_{\text {total }} & =A_{C} T_{C U E}+A_{D} T_{D U E} \\
& =\frac{\lambda_{b} \mathbb{P}\left[\operatorname{SINR} R_{C}>T_{C}\right] R_{c}(1-\eta) N k+\lambda_{D} \mathbb{P}\left[\operatorname{SINR}_{D}>T_{D}\right] R_{D} N_{D} k}{\lambda_{C}+\lambda_{D}}
\end{aligned}
$$

Finally, the throughput maximization problem is converted into an optimal spectrum allocation problem with the parameter $\eta$. Note that $\eta N$ must be an integer, the optimal spectrum allocation parameter $\eta^{*}$ can be calculated by

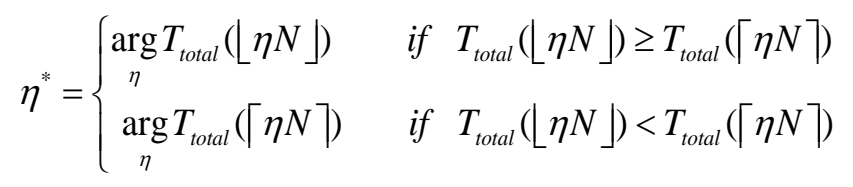

where $\lceil x\rceil$ is the smallest integer value which is larger than $x$ and $\lfloor x\rfloor$ is the biggest integer value which is smaller than $x$.

\section{Cluster-based Multi-channel Assignment}

In the previous section, we have investigated the throughput maximization problem in the case that channels are randomly assigned to D2D communications. Unfortunately, the critical interferences between nearby DUEs are not considered. In this section, we present the detail of the cluster-based multi-channel assignment algorithm to further improve the network performance. The main idea is to avoid nearby DUEs share the same channels and the interference problem can be alleviated.

The D2D communications in cellular networks are unpredictable and the DUEs are arbitrary distributed in network. Therefore, assigning channels to D2D links is a challenging work when the network scale is extremely large and the D2D communications are very dense. DUEs that are closer to each other may generate asymmetrical critical interferences to each other when they occupy the same channel simultaneously. On the other hand, DUEs that are far away from each other cannot incur strong interferences due to high path loss and limited transmit power. Based on this idea, D2D communications can be gathered into a cluster when they generate critical interferences. As Fig. 4 shows, there is no critical interference between DUEs when they belong to different clusters. Therefore, we only need to assign channels to each D2D cluster independently rather than assigning channels to all D2D links in the whole network. Thus, the scale of the problem can be significantly reduced.

The proposed algorithm includes two steps, which are 1) D2D clustering, 2) multi-channel assignment. The aim of D2D clustering is to group D2D communications into clusters on the condition that D2D links in different clusters cannot impose critical interferences. The 
multi-channel assignment algorithm is applied to each D2D cluster to avoid nearby DUEs share the same channels. Thus, the interference problem can be alleviated.

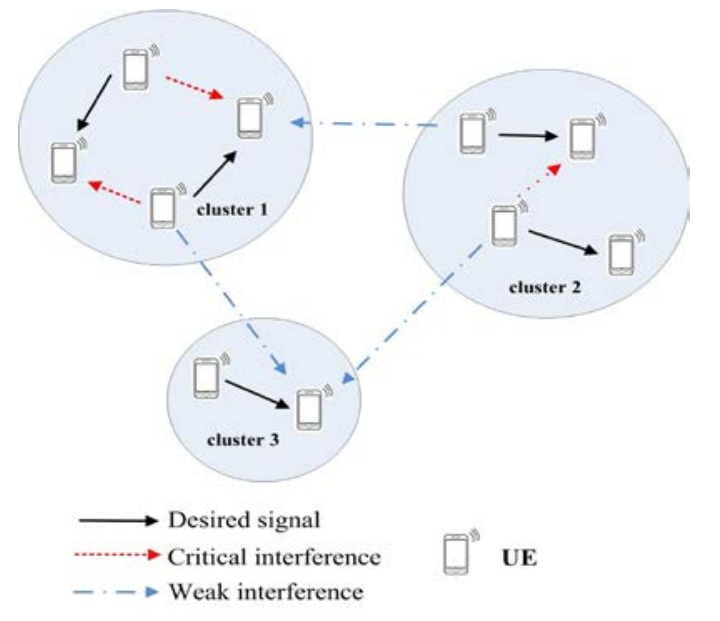

Fig. 4. D2D clustering.

\subsection{D2D Clustering}

Before clustering D2D communications into clusters, we first define the interference threshold $I_{t h}$ to characterize the critical interference or unacceptable interference between two D2D communications. The interference threshold $I_{t h}$ in this paper is defined as the maximal acceptable interference between two DUEs which participate in different D2D communications when they share a same channel. If the interference strength between two DUEs equals or exceeds $I_{t h}$, they cannot occupy any same channel. In what follows, we denote the set of the D2D communications as $\mathbf{D}$, the element $D_{j}$ denotes a typical D2D communication link. $D_{j}^{T}$ and $D_{j}^{R}$ represent the transmitter and the receiver of D2D $D_{j}$ respectively. The channel gain between $D_{j}^{T}$ and $D_{j}^{R}$ is $G_{D_{j}^{T}, D_{j}^{R}}$ and the channel gain between $D_{j}^{T}$ and $D_{j^{\prime}}^{R}$ is $G_{D_{j}^{T}, D_{j}^{R}}$ where $D_{j^{\prime}}^{R}$ is the receiver of D2D link $D_{j^{\prime}}\left(j \neq j^{\prime}\right)$. Based on the above notations and assumptions, the interference from $D_{j}$ transmitter to $D_{j^{\prime}}$ receiver can be expressed as

$$
I_{D_{j}, D_{j^{\prime}}}=P_{D} G_{D_{j}^{T}, D_{j}^{R}}
$$

and the SINR at $D_{j^{\prime}}$ receiver can be calculated as

$$
\operatorname{SINR}_{D_{j^{\prime}}}=\frac{P_{D} G_{D_{f^{\prime}}^{T}, D_{j^{R}}^{R}}}{I_{D_{j}, D_{j^{\prime}}}+\sigma^{2}}
$$

Since the DUE prescribed SINR threshold is $T_{D}$, i.e., $\operatorname{SINR}_{D_{j^{\prime}}} \geq T_{D}$. The threshold $I_{t h}$ can be calculated as

$$
I_{t h}=I_{D_{j}, D_{j}^{j}}=\frac{P_{D} G_{D_{j}^{T}, D_{j}^{R}}}{T_{D}}-\sigma^{2}
$$


When the suffered interference at a DUE exceeds the threshold, the communication outage occurs. As a result, the critical interferences in the whole network can be expressed as a $|\mathbf{D}| \times|\mathbf{D}|$ matrix I . The element $I\left(j, j^{\prime}\right)$ of matrix $\mathbf{I}$ can be calculated as

$$
I\left(D_{j}, D_{j^{\prime}}\right)=\left\{\begin{array}{cc}
1, & \text { if } I_{D_{j}, D_{j^{\prime}}^{\prime}} \geq I_{t h} \text { or } I_{D_{j^{\prime}}, D_{j}} \geq I_{t h} \\
0, & \text { otherwise }
\end{array}\right.
$$

The algorithm of clustering D2D communications into clusters is shown in Algorithm 1. In the algorithm, $\mathbf{C}$ is set to hold all the D2D communication clusters and $N_{C}$ is a variable to record the number of clusters. The algorithm searches all the unacceptable interference in the network. If two D2D communications generate unacceptable interference, we always group them into a cluster. Note that $I_{t h}$ is the maximal acceptable interference since it considers the case that there only two D2D communications share the same channel. The algorithm outputs the number of clusters $N_{C}$ and the set of clusters $\mathbf{C}$ at last.

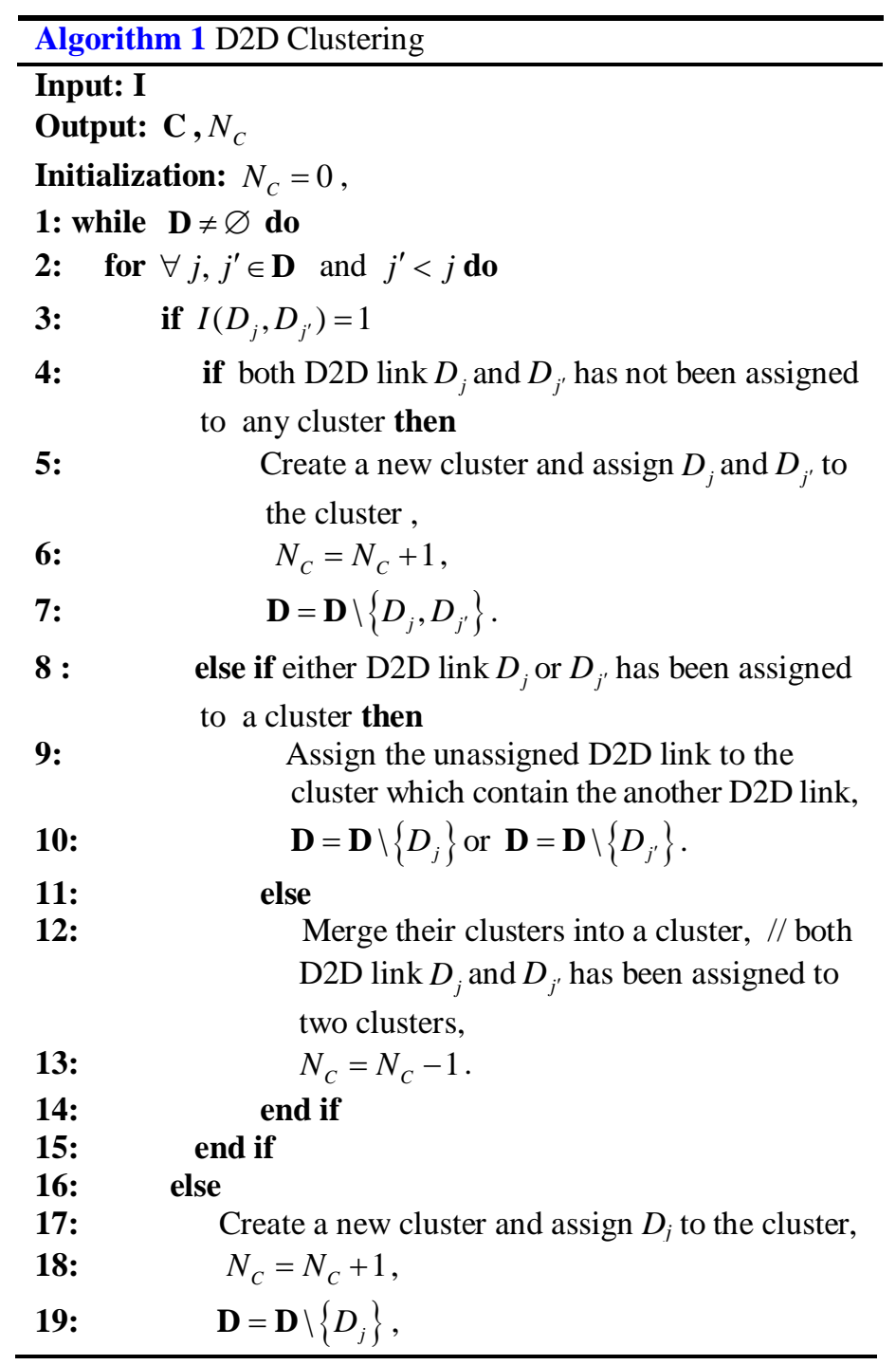




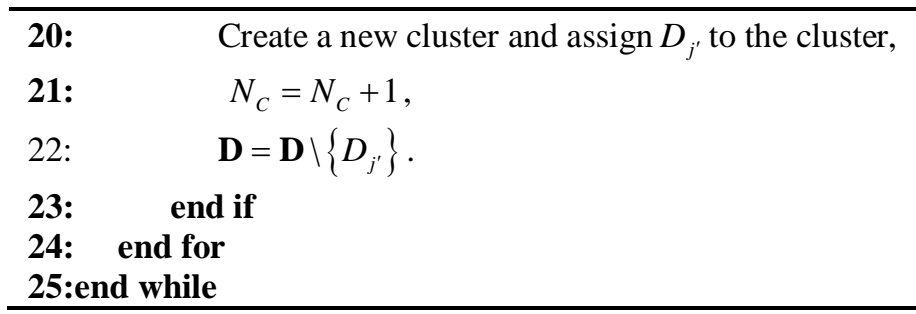

Note that as the value of $I_{t h}$ increases, more elements of $\mathbf{I}$ are equal to 1 and more clusters are merged to one. Some clusters contain only one D2D communication, i.e., DUEs of the D2D communication far away from other DUEs and cannot incur critical interference to others.

\subsection{Multi-channel assignment algorithm}

The proposed multi-channel assignment algorithm is applied to each D2D cluster independently which focus on the part of the network. Since each D2D link occupies $N_{D}$ channels which are selected from $\eta$ fraction of the system channels. It is inevitable to assign different channels to nearby two DUEs when $2 N_{D}>\eta N$. The main idea of the proposed channel assignment algorithm is trying to assign different channels to nearby DUEs, and then the interference problem can be alleviated.

The detail of the multi-channel assignment algorithm is shown in Algorithm 2. In the algorithm, $\mathbf{C}_{\mathbf{m}}$ denote a random D2D communication cluster where $\mathbf{C}_{\mathbf{m}} \in \mathbf{C}$, its element $C_{j}$ denote a D2D communication link which belongs to $\mathbf{C}_{\mathbf{m}} . \mathbf{K}$ is the set of channels used by D2D communications and $|\mathbf{K}|=\eta N$. The outcome of the algorithm is denoted by matrix $\mathbf{x}$ with dimension $\left|\mathbf{C}_{\mathbf{m}}\right| \times \eta N$, its element is a binary variable $x_{j, n}$ which denoted the channel $n$ is assigned to $C_{j}$ when $x_{j, n}=1$ and is not assigned to $C_{j}$ when $x_{j, n}=0$. The algorithm start from a randomly selected D2D communication $C_{j}$ and channel $n$ and then assign channel $n$ to $C_{j}$. $\mathbf{D}^{\prime}$ is the set of D2D communication which tries to reuse channel $n$ with $C_{j}$. In the beginning, $\mathbf{D}^{\prime}$ hold all the elements in $\mathbf{C}_{\mathbf{m}}$. The algorithm assigns channel $n$ to a typical D2D communication in $\mathbf{D}^{\prime}$ one by one and removes the D2D communications in $\mathbf{D}^{\prime}$ which generate unacceptable interference to that typical D2D communication until $\mathbf{D}^{\prime}$ is empty. After that, the algorithm randomly assigns channels to some D2D communications to satisfy the required number of channels $N_{D}$. Note that if there is only one D2D communication in a cluster, the algorithm randomly assigns channels to the D2D communication since it cannot generate critical interference to other D2D communications.

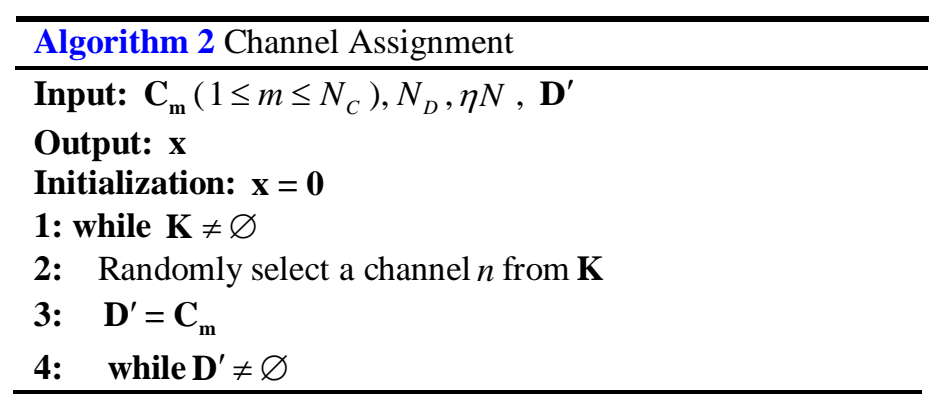




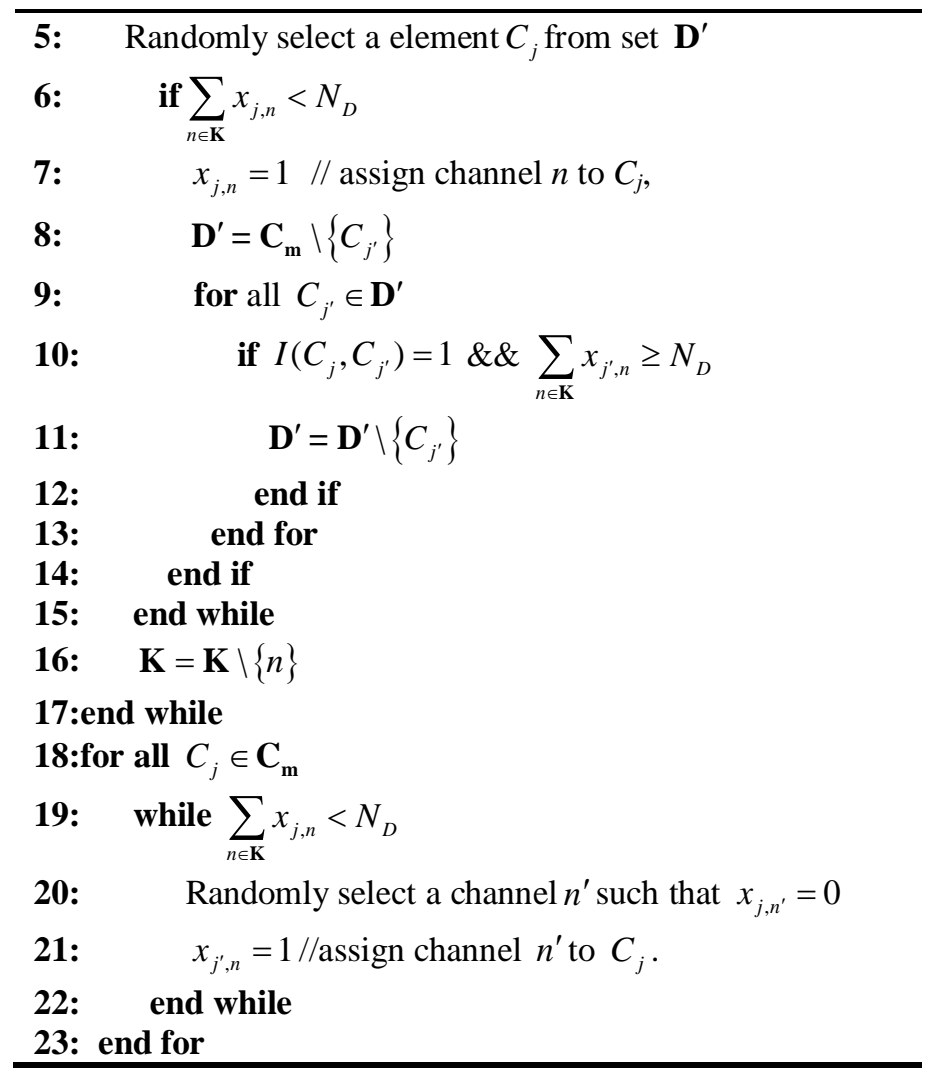

\subsection{Computational complexity analysis}

The proposed D2D clustering algorithm searchs all the element $I\left(D_{j}, D_{j^{\prime}}\right)\left(j^{\prime}<j\right)$ and groups D2D communications into clusters. The computational complexity can be calculated as $(|\mathbf{D}|-1)+(|\mathbf{D}|-2)+\cdots+1$, which is $O\left(|\mathbf{D}|^{2}\right)$. For the channel assignment algorithm, if a channel has been assigned to a typical D2D communication in $\mathbf{D}^{\prime}$, the algorithm search the other D2D communications in $\mathbf{D}^{\prime}$ which cannot generate critical interference to the typical D2D communication until $\mathbf{D}^{\prime}$ is empty. Since the number of channels is $|\mathbf{K}|$ and the number of clusters is $N_{C}$, the computational complexity can be calculated as $\left.|\mathbf{K}| \times\left(\left|\mathbf{C}_{\mathbf{m}}\right|-1\right)+\left(\left|\mathbf{C}_{\mathbf{m}}\right|-2\right)+\cdots+1\right) \times N_{C}$, which is $O\left(|\mathbf{K}|\left|\mathbf{C}_{\mathbf{m}}\right|^{2} N_{C}\right)$. In the worst case, all the D2D communications are gathered in a cluster and the overall computational complexity is $O\left(\mathbf{K}|\mathbf{D}|^{2}\right)$.

\section{Simulation Results and Analysis}

In order to evaluate the performance of our proposed algorithms, we make simulations under different number of UEs. In our settings, the transmit powers are $P_{b}=46 \mathrm{dBm}, P_{D}=8 \mathrm{dBm}$, the number of system channels is 100 , each with a bandwidth of $k=1000 \mathrm{~Hz}$. The BS density is $\lambda_{b}=1 / \pi 500^{2} \mathrm{~m}^{2}$. The path loss exponents, $\alpha_{C}$ and $\alpha_{D}$, are set as 3.5 and 4 . 
Fig. 5 shows the relationship between outage probability and D2D communication distance $b$ when the density of DUE is $5^{*} \lambda_{b}$. It is seen that at any SINR threshold, as $b$ increases, the outage probability of D2D communication also increases due to high path loss. This demonstrated that $b$ is an important parameter for D2D overlaid cellular network. We assume that $b=25 \mathrm{~m}$ in the following results, since it seems making an reasonable tradeoff between outage probability and communication distance.

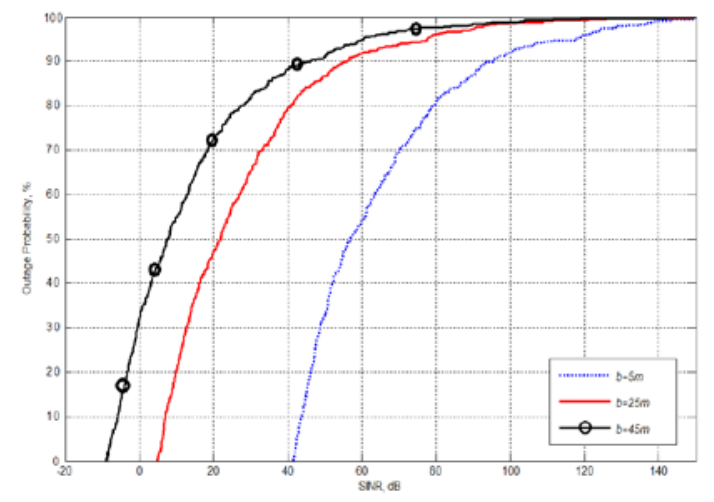

Fig. 5. The relationship between outage probability and D2D communication distance $b$.

Fig. 6 shows the outage probability under different UE density on the condition that SINR threshold $T_{C}=2 \mathrm{~dB}$ and $T_{D}=8 \mathrm{~dB}$. The x-axis indicated the CUE density and D2D transmitter density. It can be seen that no drastic changes in CUE outage probability as CUE density is increasing. This is because adding CUEs to cellulars cannot generates new interferences to current CUEs. This proves that the CUE density is independent to SINR distribution of CUEs. Different from CUEs, as the DUE transmitter density increases, the outage probability of D2D communications are also increases. This is because the probability of channel reusing is decreases and new interferences are generated by adding DUE to the network. This also shows that system throughput is actually limited by D2D interference in high density cellular networks.

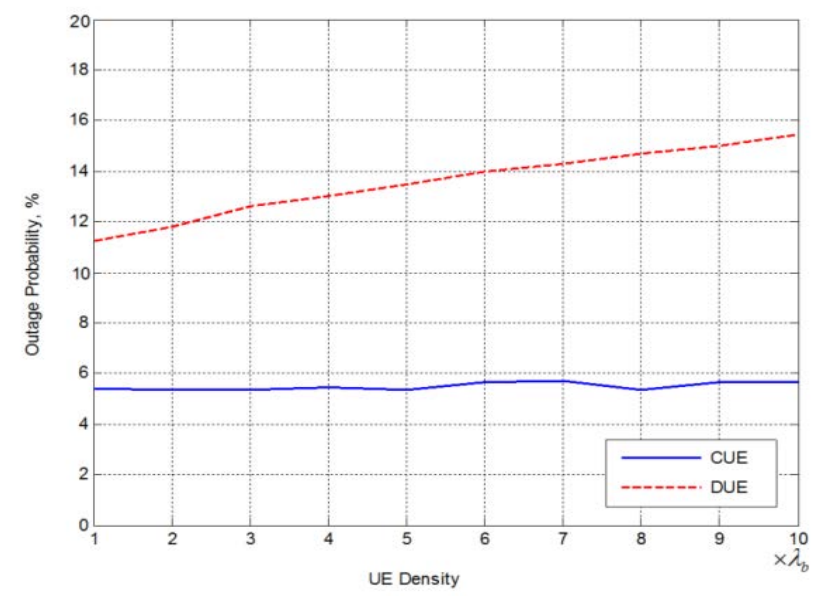

Fig. 6. Outage probability under different UE density. 
Fig. 7 shows the relationship between outage probability of D2D communications and parameter $\eta N$ on the condition that $b=25 \mathrm{~m}$ and the density of DUE is $5^{*} \lambda_{b}$. It is seen that as the value of $\eta N$ increases, the outage probability of DUE decrease. This is because as $\eta N$ increases, the probability of two DUEs that reuse a same channel is decreases, and then the D2D interference is reduced. This further confirms that the interference among DUEs can greatly impact outage probability of D2D communications.

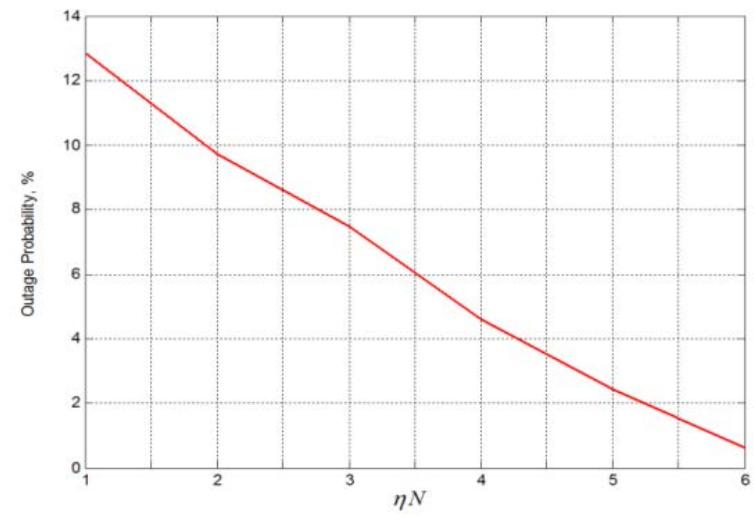

Fig. 7. The relationship between D2D outage probability and parameter $\eta N$.

In Fig. 8, the impact of the value $\eta$ on the throughput of CUEs and DUEs is analyzed. We set the density of CUEs is $5 * \lambda_{b}$ and the density of DUEs is $10 * \lambda_{b}$ as an example. Note that the same conclusion can be obtained when arbitrary density values of CUEs and DUEs are adopted. It can be seen that the throughput of CUE linearly increase with $\eta$. This is because the value of $\eta$ is independent to average ergodic rate of CUE. The throughput of DUE decreases as the value of $\eta$ increases, but the rate of decrement increases. This is because new D2D interferences are generated when the number of available channel is controlled by paprameter $\eta$.

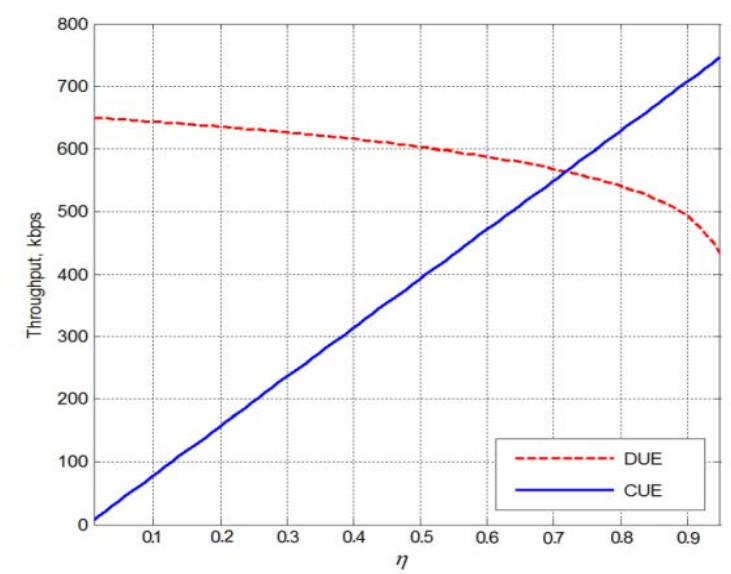

Fig. 8. UE throughput under different values of $\eta$. 
Fig. 9 shows the relaitionship between total system throughput and UE density. The value of $\eta^{*}$ is computed by equation (24). It is seen that the system achieves the maximum throughput when $\eta=\eta^{*}$. The total throughput increases as $\eta$ increases on the condition that $\lambda_{C}=\lambda_{D}=\lambda_{b}$. When $\lambda_{C}=\lambda_{D}=5 \lambda_{b}$ and $\lambda_{C}=5 \lambda_{b}, \lambda_{D}=10 \lambda_{b}$, the total system throughput increases at first but then decreases. Moreover, different UE density settings result in different value of $\eta^{*}$, i.e., the value of $\eta^{*}$ decrease when increasing DUE density. This is because an increasing number of DUE bringing D2D interferences, which needs more channels to mitigate interference, leading to a decrease in $\eta^{*}$.
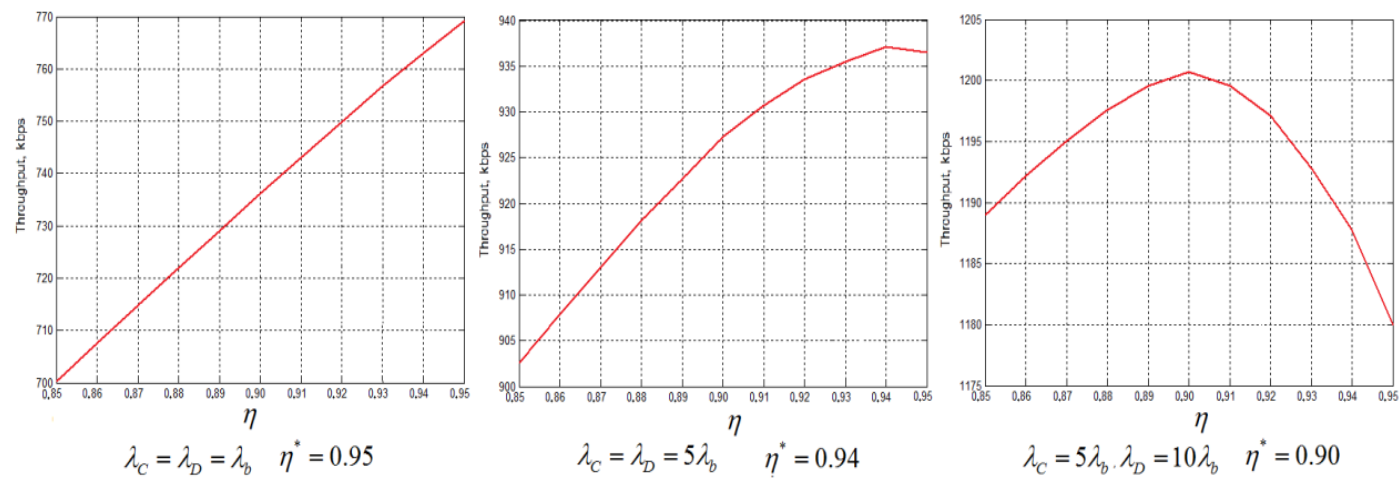

Fig. 9. The relationship between $\eta$ and throughput.

Fig.10 shows the number of clusters with varying density of DUEs. The y-axis indicates the number of D2D communications and D2D clusters. It is obvious that the number of D2D communications linear increases as the DUE density increases. This is due to the character of the PPP. We also observe that the number of cluster increases as the DUE density increases. Although more clusters sometimes merge to one, the more D2D cluster generated as the number of DUE increases since more DUEs generate critical interferences in the network.

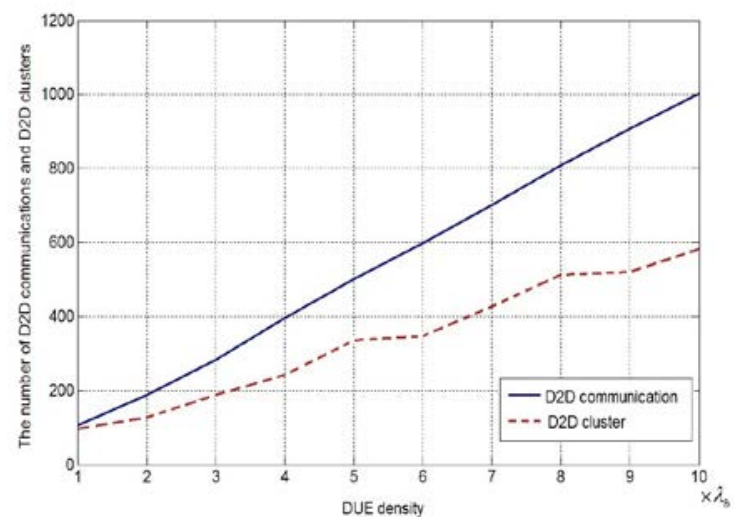

Fig. 10. The number of clusters under different density of DUE.

Fig. 11 presents the CDF of the SINR of the DUEs when the density of DUE is $10 * \lambda_{b}$. It can be seen that the proposed algorithm improves the SINR distribution when SINR is lower than $37 \mathrm{~dB}$. This is because the proposed algorithm only assigns different channels to nearby DUEs and the critical interference is alleviated. However, the other DUEs which are not generate critical interference is same to the random assignment algorithm. 


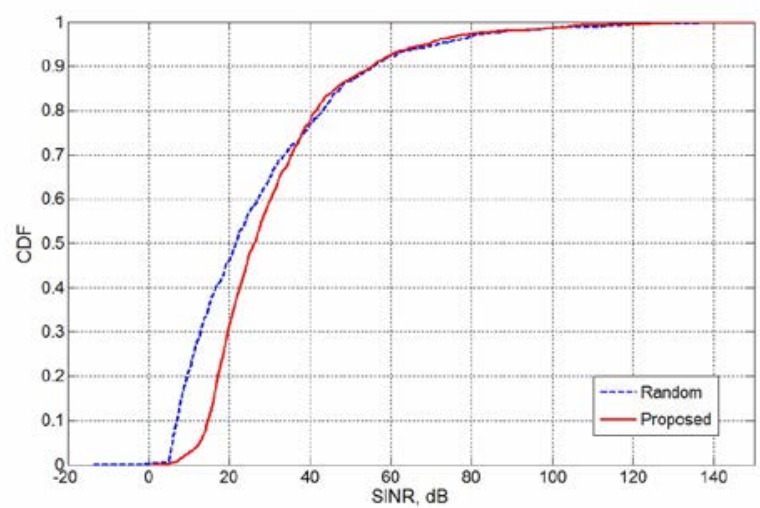

Fig. 11. CDF of SINR for D2D links.

\section{Conclusions}

This paper research on the channel assignment problem in D2D overlaid cellular networks. We first derive UE outage probability and ergodic rate by using PPP model to analyze the throughput of UEs. Then, we convert the throughput maximization problem into an optimal spectrum allocation problem with SINR constraints and solve it. Moreover, a cluster-based multi-channel assignment algorithm is proposed to mitigate the critical interference among nearby DUEs. The algorithm first cluster D2D communications into clusters to reduce the scale of the problem. Then a multi-channel assignment is proposed to mitigate interference in each cluster by assigning different channels to nearby DUEs. Simulation results show that DUE density has significantly impact on system throughput due to D2D interference. Simulation results show that our algorithm optimizes the network performance and reduces the critical among DUEs.

\section{References}

[1] M.A. Khlail, R. Hussain, and Z.U. Rehman, "Deployment of D-2-D communication, challenges and related work," in Proc. of 6th International Conference on Innovative Computing Technology, pp. 398-401, August 24-26, 2016. Article (CrossRef Link).

[2] X.H. Ge, K. Huang, C-X. Wang, X. Hong and X. Yang, "Capacity analysis of a multi-cell multi-antenna cooperative cellular network with co-channel interference," IEEE Transactions on Wireless Communications, vol. 10, no. 10, pp.3298-3309, October, 2011. Article (CrossRef Link).

[3] M. Jo, T. Maksymyuk, B. Strykhalyuk and C.-H. Cho "Device-to-device (D2D) based heterogeneous radio access network architecture for mobile cloud computing,” IEEE Wireless Communications, vol. 22, no.3, pp.50-58, June, 2015. Article (CrossRef Link).

[4] O. Bello and S. Zeadally, "Intelligent device-to-device communication in the internet of things," IEEE System Journal, vol. 10, no. 3, pp. 1172-1182, Sept. 2016. Article (CrossRef Link).

[5] T. Li, Z. Xiao, H.M. Georges, Z.N. Luo and D. Wang, "Performance analysis of co- and cross-tier device-to-device communication underlaying macro-small cell wireless networks,” KSII Transactions on Internet and Information Systems, vol. 10, no. 4, pp. 1481-1500, April, 2016. Article (CrossRef Link).

[6] J. Zhang, X. Yang, Q. Yao, X.H. Ge, M. Jo and G.Q. Mao "Cooperative energy efficiency modeling and performance analysis in co-channel interference cellular networks," The Computer Journal, vol. 56, no.8, pp.1010-1019, August, 2013. Article (CrossRef Link). 
[7] C-H. Yu, K Doppler, C.B. Ribeiro and O. Tirkkonen, "Resource sharing optimization for device-to-device communication underlaying cellular networks," IEEE Transactions on Wireless Communications, vol. 10, no. 8, pp. 2752-2763, June, 2011. Article (CrossRef Link).

[8] H. Min, J. Lee, S. Park and D. Hong, "Capacity enhancement using interference limited area for device-to-device uplink underlaying cellular networks," IEEE Transactions on Wireless Communications vol. 10, no. 12, pp. 3995-4000, October, 2011. Article (CrossRef Link).

[9] P.C. Bao and G.D. Yu, "An interference management strategy for device-to-device underlaying cellular networks with partial location information," in Proc. of IEEE: 23th International Symposium on Personal Indoor and Mobile Radio Communications, pp. 465-470, September 9-12, 2012. Article (CrossRef Link).

[10] H. Wang and X. Chu, "Distance-contrained resource-sharing criteria for device-to-device communications underlaying cellular networks,” Electronics Letters, vol. 48, no. 9, pp. 528-530, April, 2012. Article (CrossRef Link).

[11] A. Asheralieva and Y. Miyanaga, "QoS-oriented mode, spectrum, and power allocation for D2D communication underlaying LTE-A network," IEEE Transactions on Vehicular Technology, vol. 65, no. 12, pp. 9787-9800, February, 2016. Article (CrossRef Link).

[12] A. Algedir and H.H. Refai, "Adaptive D2D resources allocation underlaying (2-tier) heterogeneous cellular networks," in Proc. of IEEE International Symposium on Personal, Indoor, and Mobile Radio Communications, pp. 1-6, October 8-13, 2017. Article (CrossRef Link).

[13] J.J. Liu, S.W Zhang, H. Nishiyama and N. Kato "A Stochastic geometry analysis of D2D overlaying multi-channel downlink cellular networks," in Proc. of IEEE Conference on Computer Communications, pp. 46-54, April 26- May 1, 2015. Article (CrossRef Link).

[14] D.H. Kim and S.J. Oh, "Multi-channel-based scheduling for overlay inband device-to-device communications," Wireless Network, vol.23, no. 8, pp. 2587-2600, November, 2017. Article (CrossRef Link).

[15] G.D. Swetha and G.R. Murthy, "Selective overlay mode operation for D2D communication in dense 5G cellular networks," in Proc. of IEEE Symposium on Computers and Communications, pp. 704-709, July 3-6, 2017. Article (CrossRef Link).

[16] F. Wang, Y. Li, Z.C. Wang and Z.X. Yang, "Social-community-aware resource allocation for D2D communications underlaying cellular networks," IEEE Transactions on Vehicular Technology, vol.65, no. 5, pp. 3628-3640, June, 2015. Article (CrossRef Link).

[17] C. Ma, Y.Q. Li, H. Yu, X.Y. Gan, X.B. Wang, Y. Ren and J.J. Xu, "Cooperative spectrum sharing in D2D-enabled cellular networks," IEEE Transactions on Communications, vol. 64, no. 10, pp. 4394-4408, October, 2016. Article (CrossRef Link).

[18] X.Q. Lin, R.K. Ganti, P.J. Fleming and J.G. Andrews, "Towards understanding the fundamentals of mobility in cellular networks," IEEE Transactions on Wireless Communications, vol. 12, no. 4, pp.1686-1698, March, 2013. Article (CrossRef Link).

[19] X.H. Ge, B. Yang, J.L. Ye, G.Q. Mao, C.-X. Wang and T. Han, "Spatial spectrum and energy efficiency of random cellular networks," IEEE Transactions on Communications, vol. 63, no. 3, pp. 1019 - 1030, January 2015. Article (CrossRef Link).

[20] J.G. Andrews, F. Baccelli and R.K. Ganti, "A tractable approach to coverage and rate in cellular networks," IEEE Transactions on Communications, vol.59, no. 11, pp. 3122-3134, October, 2011. Article (CrossRef Link).

[21] X.Q. Lin, J.G. Andrews and A. Ghosh, "Spectrum sharing for Device-to-Device communication in cellular networks," IEEE Transactions on Wireless Communications, vol. 13, no. 12, pp. 6727-6740, September, 2014. Article (CrossRef Link).

[22] L.Q. Zhao, H.P. Wang and X.X. Zhong, “ On the optimal spectrum partitioning in D2D enhanced cellular sensor networks," in Proc. of IEEE International Wireless Internet Conference, pp. 197-209, December 16-17, 2017. Article (CrossRef Link).

[23] W. Bao and B. Liang, "Structured spectrum allocation and user association in heterogeneous cellular networks," in Proc. of IEEE 33th International Conference on Computer Communications, pp. 1069-1077, April 27- May 2, 2014. Article (CrossRef Link). 
[24] H.S. Dhillon, R.K. Ganti, F. Baccelli, J.G. Andrews, "Modeling and analysis of k-tier downlink heterogeneous cellular networks," IEEE Journal on Selected Areas in Communications, vol. 30, no. 3, pp. 550-560, March, 2012. Article (CrossRef Link).

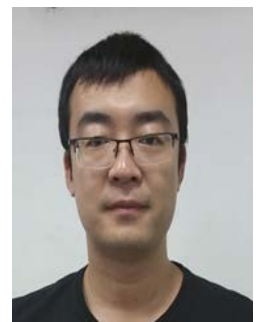

Liqun Zhao received his M.S degree in Computer Science and Technology at Harbin Institute of Technology, Shenzhen Graduate School, Shenzhen, China, in 2012. He is currently working toward a Ph.D degree with the Department of Computer Science and Technology, Harbin Institute of Technology, Shenzhen Graduate School. His general research interests include QoS and radio resource management in heterogeneous cellular networks.

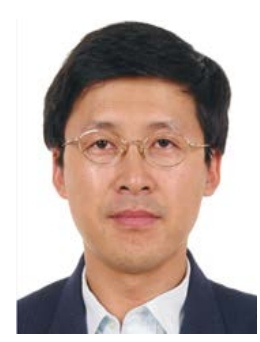

Hongpeng Wang received the Ph.D degree in Computer Science and Technology, Harbin Institute of Technology, China, in 2001. He is currently a Professor, PhD student supervisor, in School of computer science and technology, Harbin Institute of Technology Shenzhen Graduate School. His research interests include Embedded system, intelligent robot and wireless sensor networks.

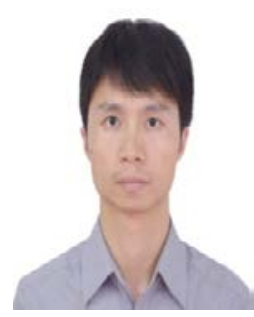

Xiaoxiong Zhong (S'14, M'16) received his Ph.D degree in Computer Science and Technology from Harbin Institute of Technology, China, in 2015. He is currently a postdoctoral fellow with the Graduate School at Shenzhen, Tsinghua University, China. His general research interests include network protocol design and analysis, internet of things and mobile computing. 